\title{
Mantle Cell Lymphoma with a Single Protruding Lesion as the Cause of Intussusception
}

\author{
Katsunori Matsueda, Tatsuya Toyokawa, Masahiro Sakata, Isao Fujita and Jouichiro Horii
}

\begin{abstract}
:
Mantle cell lymphoma (MCL) is a malignant lymphoma of the gastrointestinal tract that mostly presents as multiple lymphomatous polyposis (MLP); however, MLP with intussusception is rarely reported in MCL. Furthermore, a single protruding lesion with intussusception has never been reported in primary small intestinal MCL. A 70-year-old man presented with pain in the right lower abdomen. Computed tomography and colonoscopy revealed ileocecal intussusception. Ileocecal resection was performed. Histology and immunohistochemistry of the resected specimen showed MCL with a single protruding lesion. The patient was successfully treated with surgery alone and remains in complete remission at the three-year follow-up.
\end{abstract}

Key words: mantle cell lymphoma, protruding lesion, intussusception

(Intern Med 57: 1751-1755, 2018)

(DOI: 10.2169/internalmedicine.0199-17)

\section{Introduction}

Mantle cell lymphoma (MCL) is a non-Hodgkin's lymphoma (NHL) composed of small lymphoid cells. Approximately $6 \%$ of lymphomas are classified as MCL. MCL generally occurs in adults with a median age of 60 years and has a male predominance (1). The primary presentation of extra-nodal disease occurs in one quarter of patients and frequently involves the Waldeyer's ring and gastrointestinal tract. Primary gastrointestinal MCL accounts for $1 \%$ to $4 \%$ of primary gastrointestinal lymphomas (2). Multiple lymphomatous polyposis (MLP) is one of the most common primary gastrointestinal presentations of MCL and accounts for $9 \%$ of primary gastrointestinal lymphomas (3). It frequently occurs in the colon and small bowel, particularly in the ileum and ileocecal region. However, MCL may also present endoscopically as the protruding type, fold-thickening type, ulceration type, and superficial type throughout the gastrointestinal tract (4).

Malignant lymphoma is an uncommon cause of intussusception, causing less than $1 \%$ of all cases of intussusception (5). A single protruding lesion in the small intestine combined with intussusception has never been reported in patients with MCL. We herein report a case of primary small intestinal MCL with a single protruding lesion causing ileocecal intussusception.

\section{Case Report}

A 70-year-old previously healthy man presented with a 3day history of constant pain in the right lower abdomen, associated with nausea, without a fever or weight loss. He had no history of gastrointestinal or hematological diseases or remarkable family history. A physical examination revealed normal vital signs, a soft distended abdomen with normoactive bowel sounds, and a palpable tender mass in the right lower quadrant. His laboratory data were as follows: white blood cell count, 6,400/ $\mu \mathrm{L}$; hemoglobin level, $13.0 \mathrm{~g} / \mathrm{dL}$; Creactive protein level, $0.98 \mathrm{mg} / \mathrm{dL}$; and soluble interleukin-2 receptor level, $790 \mathrm{U} / \mathrm{m}$. Lactate dehydrogenase levels were normal. Contrast-enhanced computed tomography (CE-CT) of the abdomen showed intussusception and a mass in the terminal ileum (Fig. 1). In addition, CE-CT of the neck, chest, and pelvis revealed no lymph node enlargement or organ involvement besides in the ileocecal region.

Total colonoscopy demonstrated ileocolic intussusception and a large submucosal tumor-like protruding mass sinking into the cecum out of the terminal ileum. The mass had a smooth surface with multiple, irregularly shaped ulcerations 

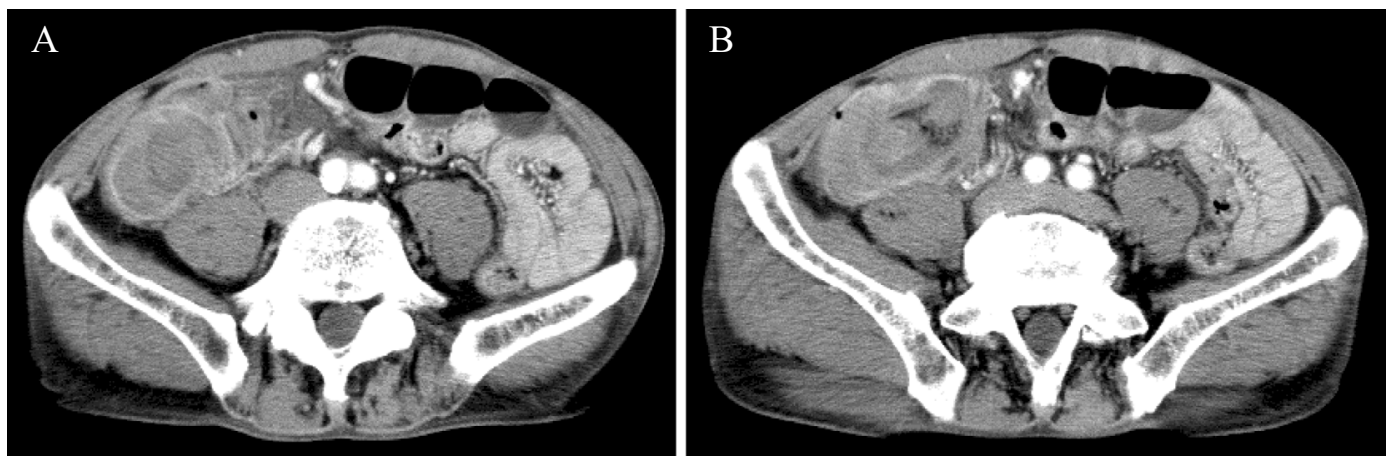

Figure 1. Contrast-enhanced computed tomography scans of the abdomen. A, B: Target sign with a fat component in the right lower quadrant.
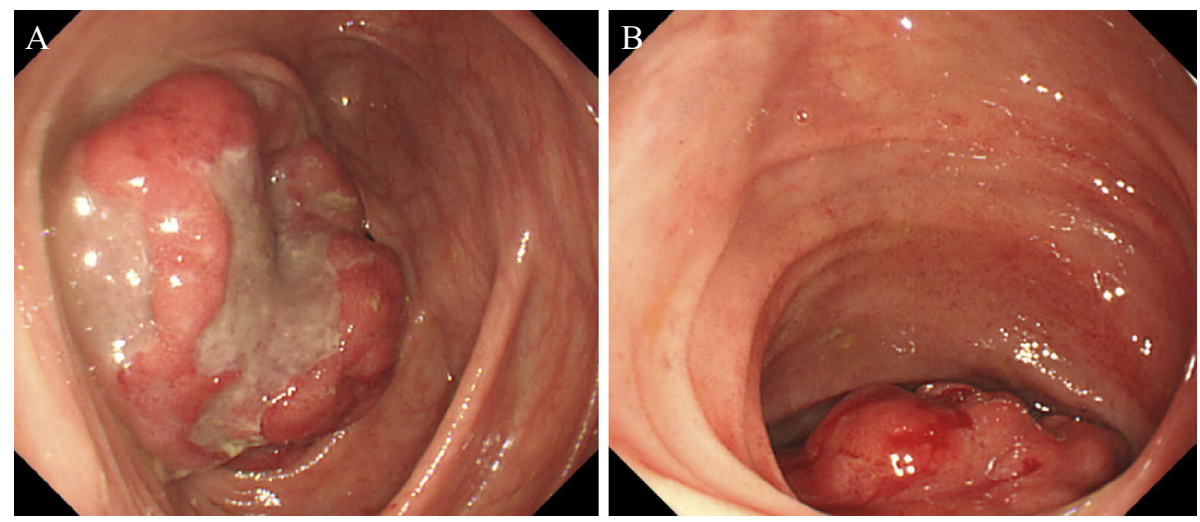

Figure 2. Endoscopic findings. A: Colonoscopy revealing ileocolic intussusception and a large submucosal tumor-like protruding mass entering the cecum and exiting the terminal ileum. B: The mass returned to the terminal ileum on colonoscopic manipulation.

and a diameter of about $50 \mathrm{~mm}$ (Fig. 2A). The mass returned to the terminal ileum on colonoscopic manipulation, resolving the intussusception without operation (Fig. 2B). Esophagogastroduodenoscopy revealed no involvement of the upper digestive tract.

Multiple biopsies of the mass showed diffuse infiltration of monotonous small to small-to-medium sized lymphoid cells (Fig. 3A and B). In addition, an immunohistochemical analysis of the biopsy tissues demonstrated positive staining for CD20 and CD5 (Fig. 3C and D); therefore, MCL was suspected. Surgery was planned to prevent intussusception from recurring.

The patient underwent ileocecal resection and ileocolic anastomosis. A gross examination of the resected specimen showed a single solid, fleshy protruding lesion, which had a cobblestone-like appearance, in the terminal ileum (Fig. 4). The lesion measured $8.8 \times 5.5 \mathrm{~mm}$. There were no other polyps in the resected specimen.

The histology of the lesion in the terminal ileum revealed a monotonous population of intermediate-sized lymphoid cells with irregular nuclear and extensive submucosal infiltration into the mucosa and muscularis propria (Fig. 5A and B). A flow cytometry analysis showed a monoclonal B-cell population with the following marker profile: surface immunoglobulin $\gamma+$, CD19+, CD20+, CD5+, CD3-,
CD10-, CD11c-, and CD23-. An immunoperoxidase analysis performed on a glass slide showed positive nuclear staining of the tumor cells for cyclin D1 (Fig. 5C). These findings were considered consistent with MCL.

Thereafter, the patient underwent staging investigations. Positron emission tomography (PET) revealed no tracer uptake. A bone marrow biopsy and chromosomal study were also performed; however, no abnormal results were observed. In addition, double-balloon enteroscopy was performed to examine the small intestine, and no other lesions were noted. Based on these findings, we diagnosed the patient with primary small intestinal MCL with a single protruding lesion. The Mantle Cell Lymphoma International Prognostic Index (MIPI) score was 3.0, and the disease was classified as stage I according to the Ann Arbor staging system.

Owing to the patient's refusal, old age, and low MIPI score, adjuvant chemotherapy was deferred, but we carefully followed up with the patient. Postoperatively, the patient never developed symptoms. Even at three years postoperatively, upper, lower, and double-balloon endoscopic examinations have demonstrated complete remission and the absence of polypoid lesions in the gastrointestinal tract. Follow-up PET showed no abnormal hypermetabolic lesions, and there was no evidence of recurrence on abdominal CT 

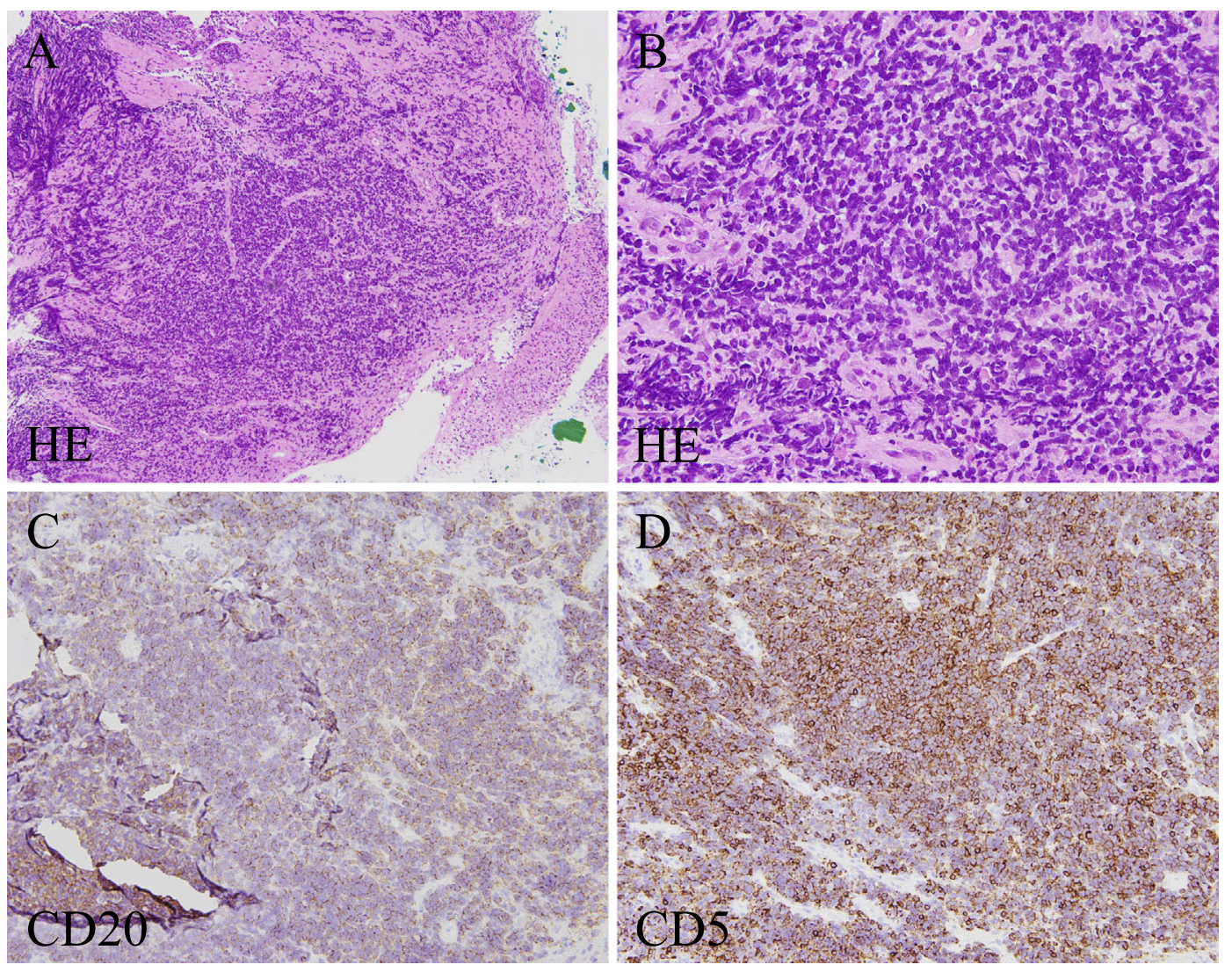

Figure 3. Pathological images of the biopsy specimens. A, B: Diffuse infiltration of monotonous small-sized to medium-sized lymphoid cells (Hematoxylin and Eosin staining; A $\times 20, B \times 200)$. C, D: Immunohistochemical staining showing positive staining for CD20 and CD5 $(\times 100)$.
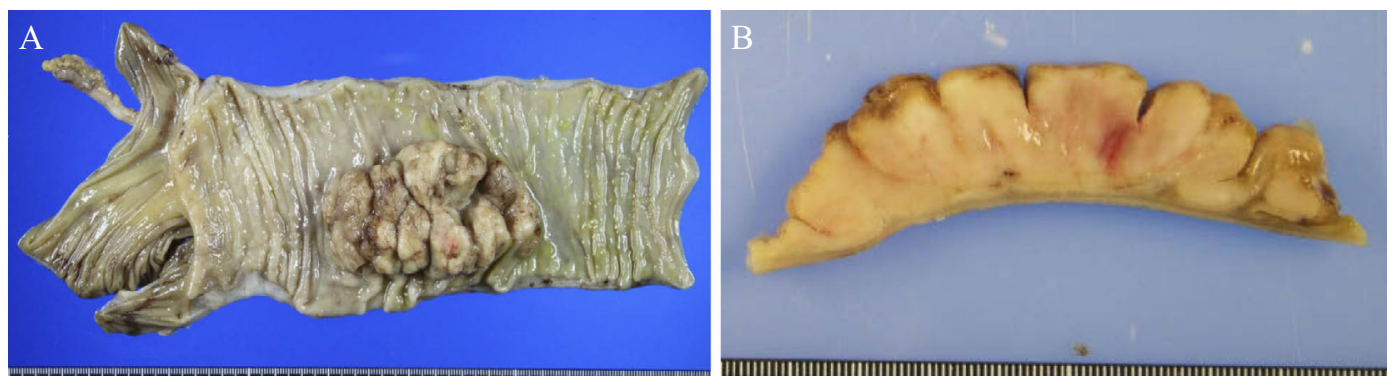

Figure 4. Surgically resected specimen. A: A single protruding lesion that acted as a leading point with a cobblestone-like appearance at the terminal ileum is shown. B: The sectioned surface of the lesion shows a whitish solid, fleshy lesion.

or endoscopic examination at the latest follow-up. The patient continues to be monitored for the development of symptoms, or changes in laboratory tests or imaging studies.

\section{Discussion}

MCL is a subtype of NHL, comprising about $7 \%$ of adult cases of NHL (6). Patients typically present at a median age of 60 years with lymphadenopathy and advance-stage disease (III or IV) (7). MCL is a well-defined entity characterized by the overexpression of cyclin D1 (6). Despite aggressive treatment, MCL has a poor prognosis, with a median overall survival of two to five years, because of its accelerated proliferation, non-specific clinical presentation, and early relapse (7).

MCL most commonly involves the gastrointestinal tract in the form of MLP. MLP accounts for 9\% of primary gastrointestinal lymphomas (3), and approximately $10 \%$ of patients with MCL present with MLP. Multiple polyps typically involve long segments of the gastrointestinal tract. The ileocecal region is most frequently involved, whereas the esophagus and anus are rarely affected $(8,9)$. Most patients with MLP will have MCL, but this presentation may also occur in follicular lymphoma and marginal zone lym- 

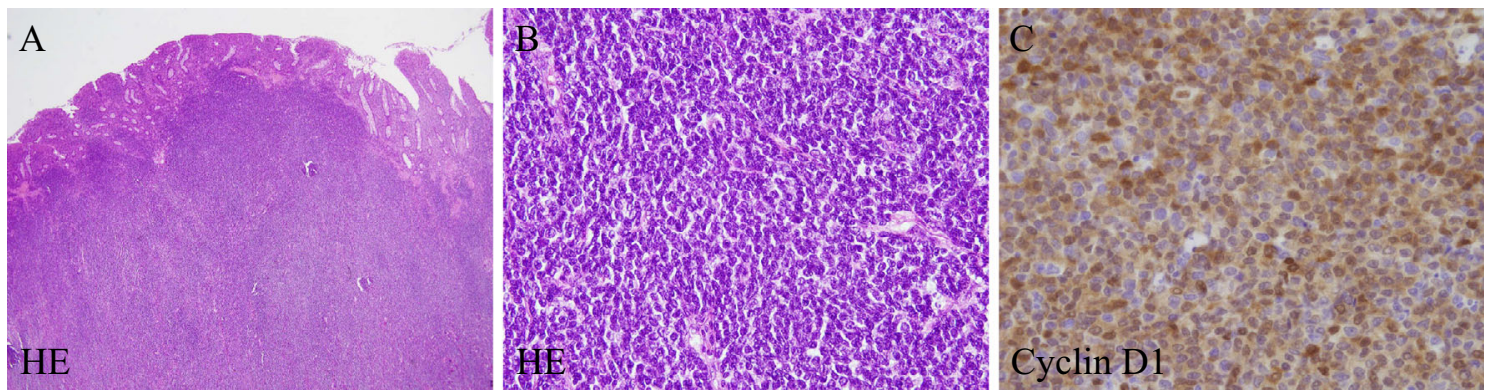

Figure 5. Pathological images of the surgically resected specimen. A: The lymphoid infiltrate fills the submucosa in the terminal ileal lesion [Hematoxylin and Eosin $(H \& E)$ staining, $\times 4$ ]. B: The cells are small to medium in size with irregular nuclei, coarse chromatin, indistinct nucleoli, and pale, scant cytoplasm. Transformed lymphocytes are absent (H\&E staining, $\times 200)$. C: Cyclin D1 is positive in most nuclei $(\times 400)$.

phoma $(8,9)$. There are also case reports of MLP in patients with adult T-cell leukemia/lymphoma (10) and angioimmunoblastic T-cell lymphoma (11).

The endoscopic appearances of primary gastrointestinal MCL are variable. In a report by Iwamuro et al. (4), intestinal involvement due to the MCL was identified in the gastrointestinal tract in $22(63 \%)$ of 35 cases. In that study, the endoscopic appearance of the intestinal lesion of MCL varied: protruding type $(n=4,18 \%)$, MLP type $(n=17,77 \%)$, and superficial type $(n=1,5 \%)$. Esmadi et al. (12) also reported that the most common endoscopic feature of primary intestinal MCL is MLP, and less commonly, it presents as protruding lesions in the intestine. In addition, Chung et al. (13) reported that all six of their patients with intestinal MCL showed multiple polyposis and no protruding-type lesions.

Instead of a common presentation of MLP, our patient's tumor consisted of a single protruding lesion, and his symptoms of intussusception were mild and never included B symptoms, such as a fever, night sweats, or weight loss. Furthermore, while MLP behaves in an aggressive manner, MCL presenting as a single protruding lesion in the small intestine has been more indolent. At more than three years since his initial diagnosis, our patient's disease remains stable. However, MCL as MLP is incurable and has a mean survival rate of three years, even with chemotherapy (14).

Intussusception is rare in adults and is usually associated with a pathological mass that acts as a lead point. In the small intestine, benign neoplasms are the most common lead points, with lipomas and hamartomatous polyps occurring in most patients. In contrast, adenocarcinomas are responsible for more than $50 \%$ of intussusceptions of the colon. In addition, because the most common lymphoma that causes intussusception is diffuse large B-cell lymphoma, MCL with a single protruding lesion in the small intestine is much rarer.

Some patients with ileus as a symptom caused by intussusception attributable to MLP in the clinical course of MCL have been reported $(13,15,16)$, but ileus being the real first symptom of intussusception attributable to primary small intestinal MCL has been rarely reported. Furthermore, to our knowledge, this is the first reported case of MCL with a single protruding lesion in the small intestine causing intussusception. Progress in the current patient suggested that complete remission may be achieved in MCL with a single protruding lesion in the small intestine through surgery without chemotherapy. This has enabled the patient to maintain a quality of life that may not have been possible with the side effects of chemotherapy.

A single protruding lesion is the atypical pattern of small intestinal involvement, and with this condition, a large mass causing intussusception, particularly involving the ileocecal region, is a rare but real complication. This case of primary small intestinal MCL with an unusual presentation adds to the spectra of clinical manifestations of small intestinal MCL. Primary small intestinal MCL should be included in the differential diagnosis of a single protruding lesion in the small intestine. Awareness of such occurrences is necessary and might help refine diagnostic and therapeutic methods for primary small intestinal MCL. Physicians should consider this possibility in any patient with a palpable tender mass in the right lower quadrant.

Therapy for MCL is based on the individual patient's risk. A simple wait-and-watch approach at the beginning is considered appropriate therapy for elderly patients or patients with a low MIPI. Chemotherapy can be started once patients develop symptoms. Initial aggressive chemotherapy with or without bone marrow transplant is recommended in young symptomatic patients. The median duration of remission is 1.5 to 3 years (17). In the current patient, the wait-andwatch approach was followed postoperatively, which was a safe option that allowed for the preservation of a good quality of life and avoidance of side effects from the early initiation of cytotoxic and biological therapies.

The authors state that they have no Conflict of Interest (COI).

\section{References}

1. Campo E, Raffeld M, Jaffe ES. Mantle-cell lymphoma. Semin Hematol 36: 115-127, 1999. 
2. Gurbuxani S, Anastasi J. What to do when you suspect gastrointestinal lymphoma: a pathologist's perspective. Clin Gastroenterol Hepatol 5: 417-421, 2007.

3. Ruskone-Fourmestraux A, Delmer A, Lavergne A, et al. Multiple lymphomatous polyposis of the gastrointestinal tract: prospective clinicopathologic study of 31 cases. Groupe D'etude des Lymphomes Digestifs. Gastroenterology 112: 7-16, 1997.

4. Iwamuro M, Okada H, Kawahara Y, et al. Endoscopic features and prognoses of mantle cell lymphoma with gastrointestinal involvement. World J Gastroenterol 16: 4661-4669, 2010.

5. Chiang JM, Lin YS. Tumor spectrum of adult intussusception. J Surg Oncol 98: 444-447, 2008.

6. Nguyen V, Nguyen B, Petris GD, Nguyen C. Education and imaging. Gastrointestinal: gastrointestinal involvement of mantle cell lymphoma. J Gastroenterol Hepatol 27: 617, 2012.

7. Weisenburger DD, Armitage JO. Mantle cell lymphoma--an entity comes of age. Blood 87: 4483-4494, 1996.

8. Kodama T, Ohshima K, Nomura K, et al. Lymphomatous polyposis of the gastrointestinal tract, including mantle cell lymphoma, follicular lymphoma and mucosa-associated lymphoid tissue lymphoma. Histopathology 47: 467-478, 2005.

9. Hashimoto Y, Nakamura N, Kuze T, Ono N, Abe M. Multiple lymphomatous polyposis of the gastrointestinal tract is a heterogenous group that includes mantle cell lymphoma and follicular lymphoma: analysis of somatic mutation of immunoglobulin heavy chain gene variable region. Hum Pathol 30: 581-587, 1999.

10. Hokama A, Tomoyose T, Yamamoto Y, et al. Adult T-cell leukemia/lymphoma presenting multiple lymphomatous polyposis.
World J Gastroenterol 14: 6584-6588, 2008.

11. Goenka MK, Vaiphei K, Nagi B, Sriram PV, Joshi K, Kochhar R. Angioimmunoblastic lymphadenopathy: an etiology for gastrointestinal lymphomatous polyposis. Am J Gastroenterol 91: 12361238, 1996.

12. Esmadi M, Ahmad DS, Duff DJ, Hammad HT. Mantle cell lymphoma of the colon. Endoscopy 46 (Suppl): E126-E127, 2014.

13. Chung $\mathrm{HH}$, Kim $\mathrm{YH}$, Kim $\mathrm{JH}$, et al. Imaging findings of mantle cell lymphoma involving gastrointestinal tract. Yonsei Med J 44: 49-57, 2003.

14. Vignote ML, Chicano M, Rodriguez FJ, et al. Multiple lymphomatous polyposis of the GI tract: report of a case and review. Gastrointest Endosc 56: 579-582, 2002.

15. Sucker C, Klima KM, Doelken G, Heidecke CD, Lorenz G, Stockschlaeder M. Unusual sites of involvement in non-Hodgkin's lymphoma: case 3. Intussusception as a rare complication of mantle-cell lymphoma. J Clin Oncol 20: 4397-4398, 2002.

16. Grin A, Chetty R, Bailey D. Mantle cell lymphoma as a rare cause of intussusception: a report of 2 cases. Ann Diagn Pathol 13: 398401, 2009.

17. Vose JM. Mantle cell lymphoma: 2012 update on diagnosis, riskstratification, and clinical management. Am J Hematol 87: 604$609,2012$.

The Internal Medicine is an Open Access article distributed under the Creative Commons Attribution-NonCommercial-NoDerivatives 4.0 International License. To view the details of this license, please visit (https://creativecommons.org/licenses/ by-nc-nd/4.0/).

(C) 2018 The Japanese Society of Internal Medicine Intern Med 57: 1751-1755, 2018 